\title{
Perfil antigénico en fase aguda y crónica de toxoplasmosis en embarazadas por la técnica de Western Blot
}

\author{
Idelina González Achar ${ }^{1,2}$, Cecilia González Vatteone $^{2}$, Ivalena Arévalo de Guillén ${ }^{1}$, María Mercedes \\ Carpinelli ${ }^{1}$, Teresa Meza ${ }^{1}$, Laura Aria ${ }^{1}$, Alejandra Rojas ${ }^{1}$, Belén Infazón ${ }^{1}$, ${ }^{*}$ María Eugenia Acosta ${ }^{1}$ \\ ${ }^{1}$ Universidad Nacional de Asunción- Instituto de Investigaciones en Ciencias de la Salud. Paraguay \\ ²Universidad Nacional de Asunción- Facultad de Ciencias Químicas. Paraguay
}

Cómo referenciar este artículo/

How to reference this article:

\begin{abstract}
González Achar I, González Vatteone C, Arévalo de Guillén I, Carpinelli M, Meza T, Aria L, et al. Perfil antigénico en fase aguda y crónica de toxoplasmosis en embarazadas por la técnica de Western Blot. Mem. Inst. Investig. Cienc. Salud. 2018; 16(3): $35-43$
\end{abstract}

\section{R E S U M E N}

La toxoplasmosis es una enfermedad endémica con prevalencia mundial variable, cuyo agente causal es el parásito Toxoplasma gondii. Para un diagnóstico certero de la infección por T. gondii son necesarias combinaciones de métodos serológicos. Estudios recientes han reportado que la técnica de Western Blot permite evidenciar proteínas antigénicas como marcadores de la infección, así como ciertos perfiles proteicos como posibles indicadores de las fases de la infección, aguda y crónica. El objetivo del estudio fue identificar el perfil antigénico específico asociado a las diferentes fases de la toxoplasmosis. Fueron incluidos en el estudio 55 sueros de embarazadas con toxoplasmosis, diferenciados en fase aguda y crónica de la enfermedad por medio del método de ELISA de Avidez de IgG. Mediante el método de Western Blot se observó que las proteínas antigénicas p35, p43, p45, p56 y p107 fueron reconocidas por el $20-60 \%$ de los sueros de pacientes en fase aguda, mientras que p65, p95, p98 y p113 fueron reconocidas por el 17-35\% de sueros de pacientes en fase crónica. Se observó que seis proteínas antigénicas, p32, p38, p41, p48, p59 y p72, fueron reconocidas por más del $60 \%$ de los sueros de pacientes tanto en fase aguda como crónica. Los resultados obtenidos sugieren que estas seis proteínas podrían ser consideradas como marcadores diagnósticos de la enfermedad.

Palabras clave: toxoplasma gondii, western Blot, perfil antigénico.

\section{Antigenic profile in the acute and chronic phase of toxoplasmosis in pregnant women using the Western Blot technique}

A B S T R A C T

Toxoplasmosis is an endemic disease with variable global prevalence, being the causative agent the parasite Toxoplasma gondii. For an accurate diagnosis of a $T$. gondii infection, combinations of serological methods are required. Recent studies have reported that the Western Blot technique allows the detection of antigenic proteins as markers of infection, as well as certain protein profiles as possible indicators of acute and chronic phases of infection. The objective of the study was to identify the specific antigenic profile associated with different phases of toxoplasmosis. Fifty five sera from pregnant women with toxoplasmosis were included in the study, differentiated in acute and chronic phase of the disease by an IgG Avidity ELISA. By using the Western Blot method it was observed that antigenic proteins p35, p43, p45, p56 and p107 were recognized by $20-60 \%$ of sera from patients in acute phase, while p65, p95, p98 and p113 were recognized by $17-35 \%$ of sera from patients in chronic phase. It was observed that six antigenic proteins, p32, p38, p41, p48, p59 and p72, were recognized by more than $60 \%$ of sera from patients in both acute

Fecha de recepción: noviembre 2018. Fecha de aceptación: diciembre 2018

*Autor correspondiente: María Eugenia Acosta de Hetter. Universidad Nacional de Asunción- Instituto de Investigaciones en Ciencias de la Salud. Paraguay

Email: maruhetter@yahoo.com.mx 
and chronic phases. The results obtained in this study suggest that these six proteins could be considered as diagnostic markers of the disease.

Keywords: Toxoplasma gondii, Western Blot, antigenic profile.

\section{INTRODUCCIÓN}

La toxoplasmosis es una zoonosis, cuya prevalencia mundial varía ampliamente entre regiones geográficas ${ }^{(1)}$. En Sudamérica, la prevalencia de anticuerpos de tipo IgG anti $T$. gondii varía ampliamente, por ejemplo es $55,9 \%$ en Chile ${ }^{(2)}, 70-81 \%$ en Brasil $^{(3)}, 21,2 \%$ en Argentina $^{(4)}$ y en Paraguay $63-84 \%$ en una población adulta ${ }^{(5,6)}$. Estas variaciones de prevalencias a nivel mundial están relacionadas con las condiciones ambientales, socioeconómicas y culturales como los hábitos alimenticios y la higiene; así como la susceptibilidad del hospedador ${ }^{(1,7)}$.

La infección por T. gondii adquirida por individuos inmunocompetentes generalmente es benigna y asintomática, sin embargo, representa una causa importante de morbilidad y mortalidad en pacientes inmunocomprometidos ${ }^{(8,9)}$. Cuando una mujer adquiere la infección primaria durante la gestación los parásitos pueden transmitirse verticalmente (desde la placenta al feto); por lo que las mujeres gestantes que cursan con una infección aguda por T. gondii pueden dar a luz a bebes con toxoplasmosis congénita ${ }^{(10-12)}$.

La infección producida por T. gondii consta de dos fases: aguda y crónica. El control de la respuesta humoral del huésped permite realizar el diagnóstico diferencial, debido a que la producción de anticuerpos específicos anti-T. gondii se correlaciona con el curso de la infección ${ }^{(13,14)}$.

Los métodos serológicos son los más empleados en el diagnóstico de toxoplasmosis, empleándose una combinación de los mismos para la distinción entre la fase aguda y crónica. Actualmente, para la detección de anticuerpos de tipo IgM e IgG y/o la demostración de seroconversión (aumento del título de anticuerpos) se emplean los ensayos de ELISA (enzyme-linked immunosorbent assay o ensayo por inmunoabsorción ligado a enzimas), QLA (Quimioluminiscencia), IFI (Inmunofluorescencia Indirecta) $(7,15,16)$. Además se realizan ensayos de avidez de IgG, los cuales permiten diferenciar los anticuerpos IgG de baja avidez, producidos en una etapa temprana de la infección de aquellos con una mayor fuerza de unión (alta avidez) que reflejan una infección latente o crónica ${ }^{(17)}$.

En Paraguay se han realizado estudios de seroprevalencia y avidez de los anticuerpos en diferentes poblaciones, sin embargo sobre las proteínas antigénicas en nuestra población fue poco estudiada. El método de Western Blot (WB) es una técnica que se basa en inmunotransferencia de proteínas del parásito o virus que permite cuantificar proteínas específicas y comparar perfiles antigénicos. Se ha utilizado para el diagnóstico confirmatorio de muchas enfermedades, el más conocido el HIV ${ }^{(18)}$. En la toxoplasmosis esta técnica se ha empleado para demostrar la presencia de marcadores antigénicos que permiten distinguir entre la fase aguda y crónica de la toxoplasmosis ${ }^{(19-21)}$. En los últimos años se ha aplicado para confirmación de toxoplasmosis congénita, y se estimó una sensibilidad del WB del 72 al $85 \%$ al tercer mes de vida y al combinarlo con serología la sensibilidad aumentó a $94 \%$ con especificidad de $100 \%{ }^{(22)}$. Es importante conocer las proteínas antigénicas más frecuentes de los pacientes con toxoplasmosis a nivel nacional, ya que podría ser factible para el desarrollo de otros métodos de diagnóstico que faciliten la confirmación de la enfermedad. Es por esto que este trabajo tuvo por objetivo identificar el perfil antigénico asociado a embarazadas con toxoplasmosis en fase aguda y crónica de la enfermedad.

\section{MATERIALES Y MÉTODOS Sueros humanos}

Los 55 sueros de mujeres embarazadas empleados en este estudio fueron codificados y almacenados en la seroteca del Dpto. de Producción del Instituto de Investigaciones en Ciencias de la Salud, todos obtenidos con previo consentimiento informado y una encuesta de antecedentes serológicos de IgG e IgM en el momento de la toma de muestra. Se consideraron agudos los valores menores de $45 \%$ y crónicos a valores mayores de $50 \%$, fueron diagnosticados por medio del ELISA de Avidez IgG (TOXOPLUS-IICS, Paraguay) relacionando los resultados con los antecedentes de cada paciente. 
Se incluyeron 27 sueros de pacientes con toxoplasmosis aguda con avidez $<45 \%$; y datos de IgG e IgM positiva y 28 sueros de pacientes con toxoplasmosis crónica con avidez $>50 \%$, con IgG positiva e IgM negativa.

\section{Controles}

Se emplearon como control positivo y control negativo un pool de sueros de pacientes con diagnostico positivo y negativo para toxoplasmosis, respectivamente, los cuales fueron testados por medio de los métodos de diagnóstico de ELISA de Avidez IgG e Inmunofluorescencia Indirecta.

\section{Producción de antígeno soluble de Toxoplasma gondii para el WB}

Fueron inoculados por vía intraperitoneal $0,1 \mathrm{~mL}$ de una suspensión de $2 \times 107$ taquizoítos/ $\mathrm{mL}$ a cuatro ratones hembras albinos tipo suizo de un mes de edad. A los cuatro días de la inoculación, se sacrificaron los ratones por el método de eutanasia con dióxido de carbono. Se inyectaron $3 \mathrm{~mL}$ de solución fisiológica más gentamicina $(400 \mathrm{mg} / \mathrm{mL})$ en la cavidad intraperitoneal, se aspiró, se recogió el fluido intraperitoneal en tubos centrifuga de $50 \mathrm{~mL}$ y se observó entre lámina y laminilla, con aumento de 40x en el microscopio óptico (Olympus). Luego se inoculó $0,1 \mathrm{~mL}$ del líquido intraperitoneal realizando varios pasajes hasta obtener campo lleno de parásitos con aumento de 40x en el microscopio óptico.

Para la producción del antígeno se realizó un pool de los lavados intraperitoneales de todos los ratones infectados del último pasaje con campo lleno de parásitos, se centrifugó a 3500 $\mathrm{rpm}$ a $4^{\circ} \mathrm{C}$ por 20 minutos, se eliminó el sobrenadante y el precipitado se resuspendió con $5 \mathrm{~mL}$ de solución fisiológica más gentamicina $(400 \mathrm{mg} / \mathrm{mL})$, posteriormente se adicionó un agente antiproteolítico para su conservación a $-20{ }^{\circ} \mathrm{C}$. La ruptura de los taquizoítos se realizó mediante doce ciclos de sonicación en baño de hielo. Se centrifugó a una velocidad de $3500 \mathrm{rpm}$ por 20 minutos a $4^{\circ} \mathrm{C}$. La concentración de proteínas presentes en el sobrenadante se determinó por el método de Bradford ${ }^{(23)}$, empleando como patrón albúmina de suero bovino (ASB) para la obtención de una curva patrón y posterior cálculo de la concentración de las proteínas solubles del antígeno preparado.

\section{Electroforesis en gel de poliacrilamida con dodecil sulfato de Sodio (SDS-PAGE) e inmunotransferencia.}

Las proteínas solubles de taquizoítos de la cepa RH de $T$. gondii se separaron por de electroforesis (SDS-PAGE) descrita por Laemmli ${ }^{(24)}$ con algunas modificaciones. Se utilizó una concentración del gel de poliacrilamida de $5 \%$ en su fracción concentradora y $12 \%$ en la fracción separadora. La corrida electroforética se realizó probando tiempos de corrida de 90 minutos, y aplicando corriente constante de 40mA (miliamperios).

Se mezclaron $200 \mathrm{uL}$ de la suspensión de proteínas solubles $(3,98 \mathrm{ug} / \mu \mathrm{L})$ con $100 \mu \mathrm{L}$ de tampón de carga; la mezcla se incubó a $70^{\circ} \mathrm{C}$ por 5 minutos. Los volúmenes de carga de la muestra variaron de 8-12 $\mathrm{LL}$. Se cargaron las muestras y el marcador de peso molecular (Bio-Rad-USA) en el mismo gel para estimar el peso molecular de cada proteína antigénica. Posterior a la electroforesis, se realizó la tinción del gel con Coomasie blue.

Se realizó Western Blot según el protocolo de Towbin et al. ${ }^{(25)}$ con algunas modificaciones. Las fracciones proteicas separadas por SDS-PAGE se transfirieron a la membrana de polivinildifluoruro (PVDF) (Millipore, USA).La transferencia se realizó probando las siguientes condiciones: voltaje constante $59 \mathrm{~V}$ (voltios) y tiempos de corrida de 120 minutos. Las membranas cortadas de $0,5 \mathrm{~mm}$ de ancho fueron guardadas a $-20^{\circ} \mathrm{C}$ hasta su utilización.

\section{Inmunoblotting}

Se emplearon sueros controles positivos y negativos de un Kit de ELISA indirecto IgG (TOXO TEST IICS, Paraguay) y para determinación del perfil 55 muestras de suero siguiendo la técnica. Los sueros fueron diluidos $(1 / 20)$ con solución salina tamponada con TRIS (TBS)-leche descremada al 3\% .Se incubaron las membranas en agitación durante $1 \mathrm{~h}$ $30 \mathrm{~min}$. Se realizaron 3 lavados de 5 minutos cada uno con tampón TBS, Se incubó $1 \mathrm{~h}$ con $1 \mathrm{~mL}$ de anticuerpo secundario anti IgG humana de cabra conjugado con peroxidasa (IICS-UNA), diluido $1 / 1000$ con TBS-leche descremada al 3\%. Se realizaron 3 lavados de 5 minutos cada uno con TBS-Tween 20 al 0,05\%; posteriormente se adicionó $0,5 \mathrm{mg} / \mathrm{mL}$ del sustrato cromógeno 3,3' tetrahidrocloruro de diaminobencidina (DAB) (Sigma/Aldrich -USA) 
y peróxido de hidrógeno al $0,01 \%$ en tampón TBS. Una vez visualizadas las bandas, se detuvo la reacción con agua destilada.

\section{Determinación del perfil antigénico}

Para identificar el perfil de las proteínas antigénicas presentes en las membranas transferidas, de los 55 sueros pacientes con diagnóstico de toxoplasmosis positivo; diferenciados en agudos y crónicos por el test de Avidez IgG (TOXOPLUS-IICS, Paraguay) se utilizó el programa ImageJ Ccm (salud.ccm.net).

\section{Obtención y análisis de datos}

Para el análisis del registro fotográfico de los geles de poliacrilamida como de las membranas transferidas se utilizó el programa Image] $\mathrm{Ccm}$ (salud.ccm.net) para el análisis de las bandas con el fin de determinar los pesos moleculares con referencia al marcador (Bio-Rad-USA). Los datos fueron introducidos en una hoja de cálculo de Excel para expresar los resultados en forma de distribución de frecuencia y el cálculo de desviación estándar.

\section{Consideraciones éticas}

El trabajo de investigación fue un objetivo dentro del proyecto marco P17/2017 aprobado por el Comité Científico y Comité de Ética en la Investigación del IICS.

Si bien estos pacientes no fueron beneficiados directamente, los resultados obtenidos en cuanto al perfil antigénico servirán como base para nuevos estudios en el proyecto con beneficio directo a nuevos pacientes.

\section{RESULTADOS}

A partir de la metodología empleada se obtuvo una alta concentración de proteínas solubles de $T$. gondii de $3,98 \mathrm{mg} / \mathrm{mL}$. Las proteínas solubles de taquizoítos de Toxoplasma gondii fueron utilizados para la prueba de Western Blot y detectar marcadores antigénicos. En las proteínas presentes en el extracto de $T$. gondii separadas por medio de SDS-PAGE, se observó el patrón de bandas con un rango de peso molecular comprendido entre 15kDa y mayor a 160 kDa (Figura 1).

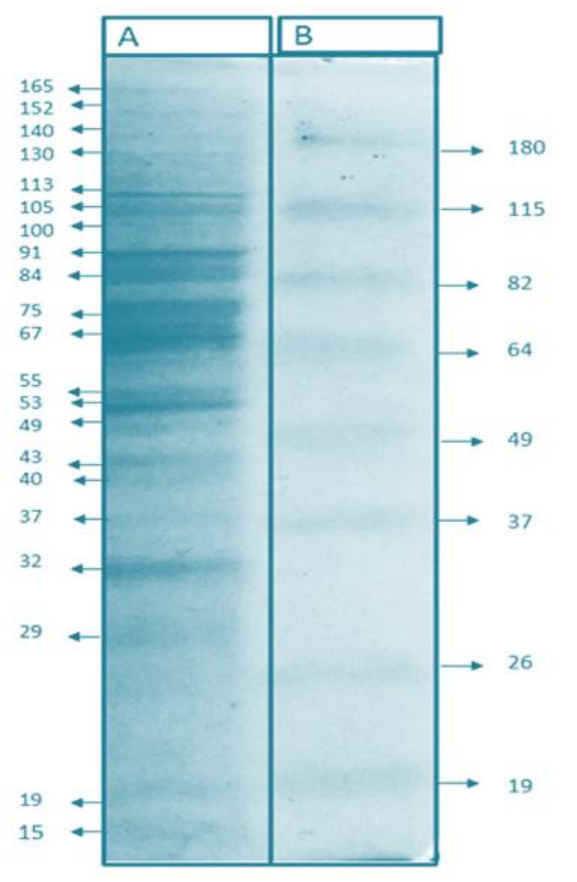

Figura 1. Patrón de Proteínas del extracto T. gondii. (A) Patrón de proteínas de la cepa RH de T. gondii de separadas por SDS-PAGE. (B) Marcador de peso molecular. Estimación de peso molecular realizado mediante el software ImageJ. (kDa)(kiloDaltons). 
En el Inmunoblotting se observó reacción positiva, evidenciada por el reconocimiento antigénico por parte de anticuerpos IgG, empleando un control positivo (A) al emplear el control negativo no se observó reacción (B). Figura 2

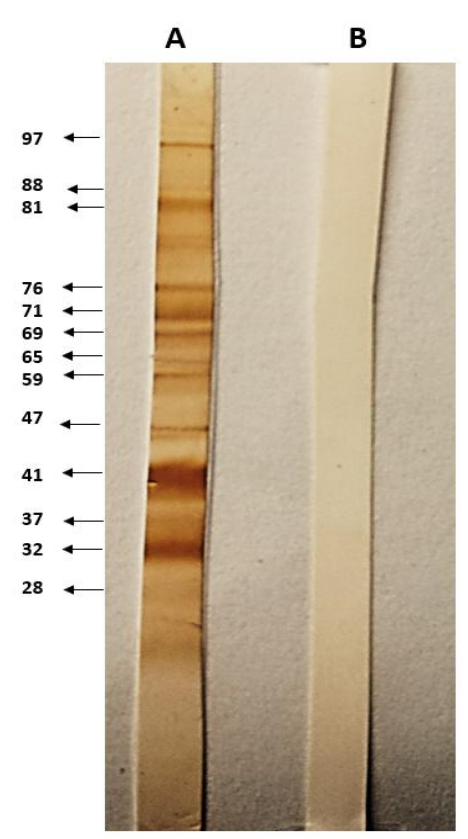

Figura 2. Inmunoblot de extractos proteicos de la Cepa RH de T. gondii. Se indican los pesos moleculares de las proteínas correspondientes a las bandas detectadas. A: reacción con control positivo. B: reacción con control negativo.

En cuanto al perfil de proteínas antigénicas del extracto proteico de la Cepa RH de $T$. gondii, los sueros de pacientes analizados reconocieron 29 bandas de proteínas antigénicas asociadas al perfil de infección aguda y 26 bandas de proteínas antigénicas asociadas al perfil de infección crónica. Los pesos moleculares de las bandas detectadas se encuentran dentro de un rango de $15 \mathrm{kDa}$ a > $115 \mathrm{kDa}$.

De las proteínas antigénicas reconocidas por anticuerpos IgG anti- $T$. gondii presentes en muestras de suero de pacientes con infección aguda, del 20 al $60 \%$ reconocieron seis bandas correspondientes a las proteínas p35, p43, p45, p56, p100 y p107 que no fueron observadas en el perfil de proteínas antigénicas de pacientes con infección crónica.

De las 26 bandas de proteínas antigénicas asociadas al perfil de infección crónica, las bandas correspondientes a las proteínas p65, p95, p98 y p113 fueron reconocidas por el $17-35 \%$ de los pacientes de manera específica. En la Figura 3 y 4 se observan que las proteínas p32, p38, p41, p48, p59 y p72 fueron reconocidas por los anticuerpos tipo IgG anti $T$. gondii en más del $60 \%$ de los 55 sueros de pacientes tanto al inicio de la infección como en la etapa crónica. 


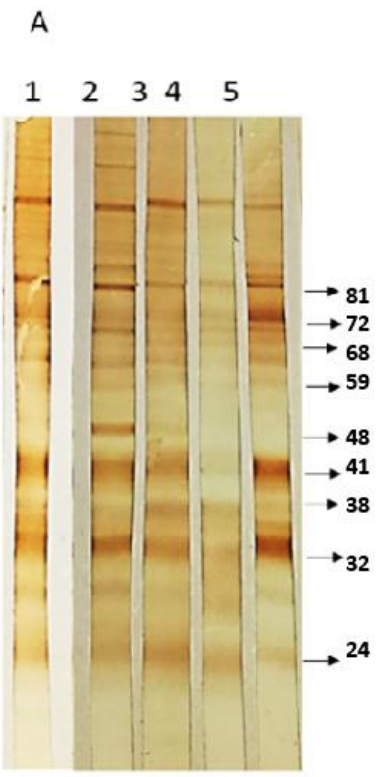

B

$\begin{array}{llllll}1 & 2 & 3 & 4 & 5 & 6\end{array}$

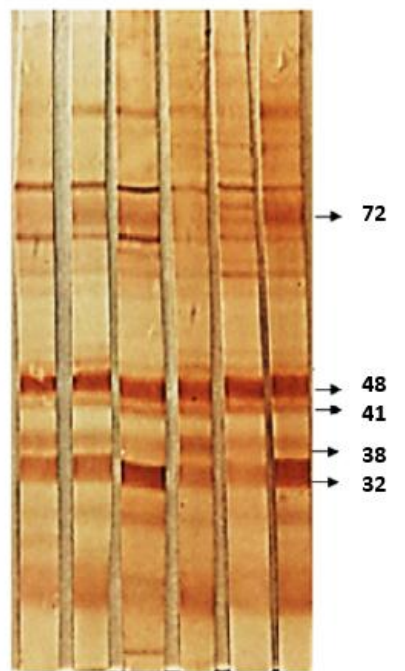

Figura 3. Perfil de proteínas antigénicas de T. gondii cepa RH. (A) Reacción positiva frente a sueros de pacientes con Toxoplasmosis aguda, (B) Reacción Positiva frente a sueros de pacientes con Toxoplasmosis crónica.

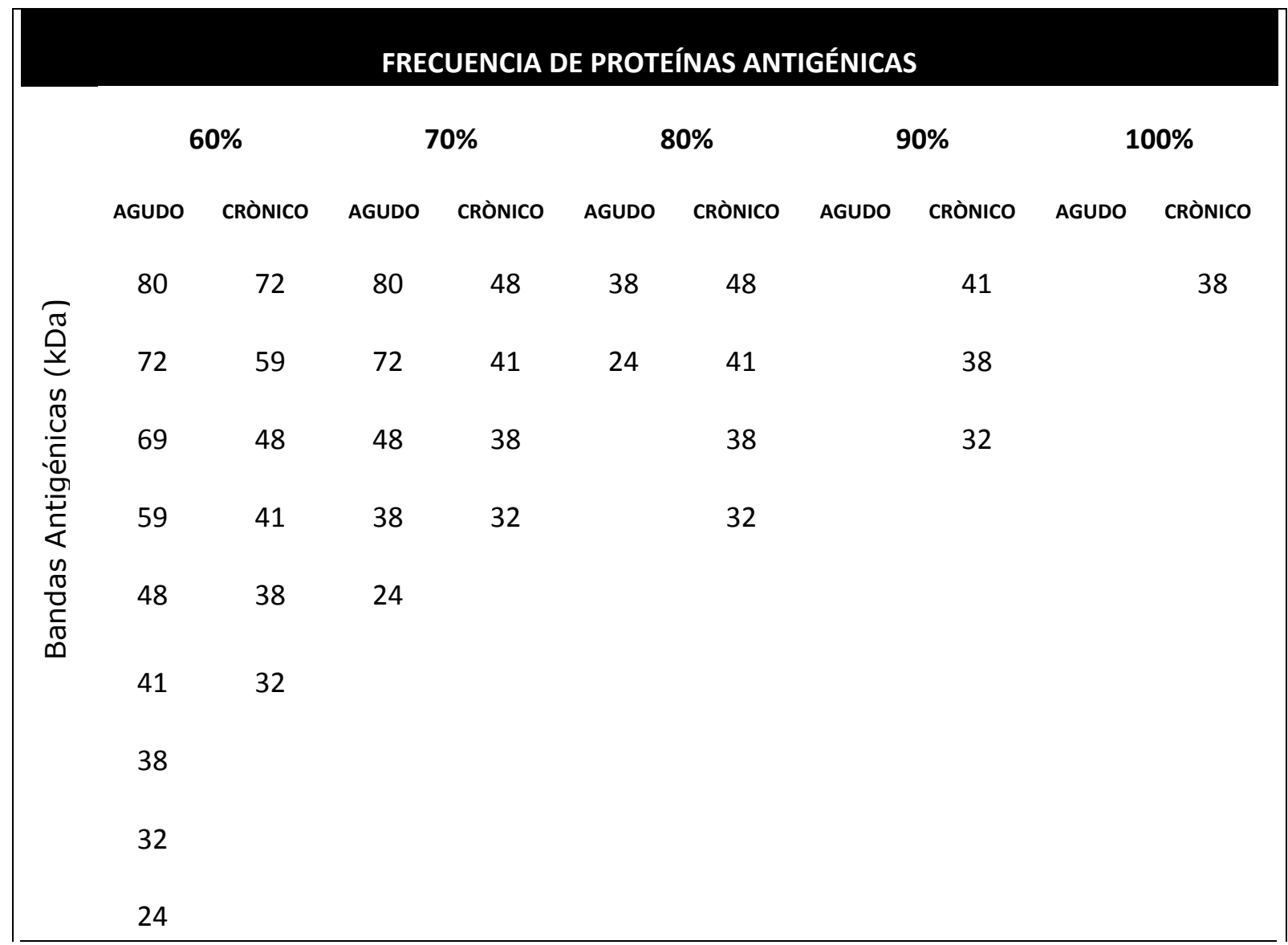

Figura 4. Frecuencia de proteínas antigénicas en kDa (kiloDaltons) reconocidas por sueros de embarazadas con infección aguda y crónica de toxoplasmosis. 


\section{DISCUSIÓN}

Para el diagnóstico confirmatorio de la toxoplasmosis, se han llevado a cabo estudios en relación al empleo del Western Blot en la detección de marcadores antigénicos específicos.

La utilización de la cepa RH de $T$. gondii en la fracción soluble para la determinación del perfil antigénico coincide con otros autores que utilizaron cepas también virulentas como GT1 obteniendo respuestas similares ${ }^{(26,27)}$. Otros autores informaron que los principales antígenos solubles estarían dentro de un rango de tamaño 20-120 kDa y así como también en un rango de $<16$ y $>116 \mathrm{kDa}$ y $<15$ y $>190 \mathrm{kDa}$ coincidiendo con el rango de proteína encontrado en nuestro estudio ${ }^{(17,27-29)}$.

Teniendo en cuenta la etapa de la infección, diversos estudios difieren con respecto a la presencia de proteínas antigénicas, las cuales varían de acuerdo al tipo de cepa utilizada para el diagnóstico. Se han reportado proteínas de fase aguda que van de 60 a $70 \mathrm{kDa}$ en cepas como RH, Ankara, Ts-4 y mayores a $85 \mathrm{kDa}$ en la cepa $\mathrm{SUBI}^{(30)}$, por lo que cada región debería estandarizar el perfil según la cepa de $T$. gondii utilizada.

En un estudio de marcadores moleculares de fase aguda y crónica de toxoplasmosis en humanos se obtuvieron resultados similares al nuestro, en el cual las proteínas p32, p41 y p72 también fueron reconocidas por anticuerpos IgG anti-T. gondii en muestras con las formas recientes y crónicas de toxoplasmosis, en tanto que p38 fue considerada como marcador de infección reciente ${ }^{(21)}$. Otros estudios han descrito proteínas detectadas por anticuerpos de tipo IgG anti- $T$. gondii de interés diagnóstico, cuyo peso molecular se encuentra comprendido entre $32-35 \mathrm{kDa}$ y $40-45 \mathrm{kDa}^{(26,31,32)}$.

En cuanto a proteínas marcadoras de infección encontradas en nuestro trabajo, se observó que las proteínas antigénicas correspondientes a p35, p43, p45, p56, p100 y p107 se asociaron al perfil antigénico de infección aguda; en tanto que las proteínas p65, p95, p98 y p113 se asociaron de manera específica al perfil de infección crónica. Las bandas que fueron visualizadas con mayor frecuencia en la población de estudio correspondieron a las proteínas con peso molecular entre $24-44,48-60$ y $69-72 \mathrm{kDa}$, de las cuales las proteínas antigénicas p32, p38, p41, p48, p59 y p72 fueron reconocidas por pacientes con infección aguda y crónica, las cuales se podrían considerar como bandas diagnósticas de la enfermedad.

Los métodos serológicos son muy utilizados para el diagnóstico y seguimiento de los pacientes con Toxoplasmosis, en especial en determinadas poblaciones como las mujeres embarazadas y los niños con sospecha de Toxoplasmosis congénita. En algunas ocasiones, las determinaciones de anticuerpos anti-Toxoplasma gondii podrían dar resultados dudosos por lo que es necesario contar con un método confirmatorio como el Western Blot, que emplea proteínas específicas y permite determinar perfiles antigénicos que evidencian la presencia de una infección reciente o la transmisión congénita en los hijos de madres que adquirieron la infección durante la gestación, por lo cual puede ser aplicado en métodos de diagnóstico complementarios aún poco utilizados a nivel nacional.

\section{REFERENCIAS BIBLIOGRÁFICAS}

1. Furtado JM, Smith JR, Belfort R, Gattey D, Winthrop KL. Toxoplasmosis: A Global Threat. J Glob Infect Dis. 2011;3(3):281-4.

2. Munoz-Zanzi C, Campbell C, Berg S. Seroepidemiology of toxoplasmosis in rural and urban communities from Los Rios Region, Chile. Infect Ecol Epidemiol [Internet]. 2016 [citado 27 de febrero de 2018];6. Disponible en: https://www.ncbi.nlm.nih.gov/pmc/articles/ PMC4788768/

3. Carmo EL do, Morais R dos APB, Oliveira AS de, Figueredo JE, Figueredo MC, Silva AV da, et al. Seroepidemiology of Toxoplasma gondii infection in the City of Novo Repartimento, Pará State, Brazil. Rev PanAmaz Saude. 2016;79-87.

4. Kaufer FJ, Carral LA, Messina MT, Schneider MV, Méndez M, Herrera $L$, et al. Prevalencia de anticuerpos anti Toxoplasma gondii en hemodonantes en la ciudad de Buenos Aires, desde 1967 a 2017. 2017;6.

5. Guerrero Hernández AC, Gómez J, Samudio M, Bolaños A, Dorantes Y, Scalamogna M, et al. Prevalence of Toxoplasma retinochoroidal scars in a Paraguayan population. Mem Inst Investig En Cienc Salud. 2016;14(1):78-85.

6. Romero DA, González-Vatteone C, Guillen I de, Aria L, Meza T, Rojas A, et al. Seroprevalence and associated risk factors of toxoplasmosis among women in reproductive age who attended district Hospital of Lambaré, Paraguay. Mem Inst Investig En Cienc Salud. 2017;15(3):83-8.

7. Mimica F, Muñoz-Zanzi C, Torres M, Padilla O. Toxoplasmosis, zoonosis parasitaria prevalente en Chile: recuento y desafíos. Rev Chil Infectol. 2015;32(5):541-9. 
8. Walle $\mathrm{F}$, Kebede $\mathrm{N}$, Tsegaye A, Kassa $\mathrm{T}$. Seroprevalence and risk factors for Toxoplasmosis in HIV infected and noninfected individuals in Bahir Dar, Northwest Ethiopia. Parasit Vectors. 2013;6:15.

9. Efunshile AM, Elikwu CJ, Jokelainen $P$. Toxoplasmosis - Awareness and knowledge among medical doctors in Nigeria. PLoS ONE [Internet]. 19 de diciembre de 2017 [citado 27 de febrero de 2018];12(12). Disponible en: https://www.ncbi.nlm.nih.gov/pmc/articles/ PMC5736225/

10. Durlach R, Kaufer $F$, Carral L, Freuler C, Ceriotto $M$, Rodriguez $M$, et al. [Argentine Consensus of Congenital Toxoplasmosis]. Medicina (Mex). 2008;68:75-87.

11. Thellaeche Cortez C, Cervantes Mederos M, Miahuchi D. Toxoplasmosis congénita, reporte de casos. Rev Soc Boliv Pediatría. 2014;53(1):12-6.

12. Hill D, Dubey JP. Toxoplasma gondii: transmission, diagnosis and prevention. Clin Microbiol Infect. 2002;8(10):634-40.

13. Millar PR, de Moura FL, Bastos OMP, de Mattos DPBG, Fonseca ABM, Sudré AP, et al. Toxoplasmosis-Related knowledge among pregnant and postpartum women attended in public health units in Niterói, Rio De Janeiro, Brazil. Rev Inst Med Trop São Paulo. 2014;56(5):433-8.

14. Cofré F, Delpiano L, Labraña $Y$, Reyes $A$, Sandoval A, Izquierdo G. Síndrome de TORCH: Enfoque racional del diagnóstico y tratamiento pre y post natal.: Recomendaciones del Comité Consultivo de Infecciones Neonatales Sociedad Chilena de Infectología, 2016. Rev Chil Obstet Ginecol. 2017;82(2):171-211.

15. Cortes JA, Gómez JE, Silva PI, Arévalo $L$, Rodríguez IA, Álvarez MI, et al. Clinical practice guideline. Integral Care Guidelines for the prevention, early detection and treatment of pregnancy, childbirth and puerperium complications: Section on toxoplasmosis in pregnancy. Infectio. 2017;102-16.

16. Goya Batista $Y$, Cobos Valdes D, Sánchez Artigas R, Miranda Cruz A, Torres Ponce Z, Labañino Mulet N. Comparación de dos métodos serológicos para el diagnóstico de anticuerpos en neonatos. Rev Cuba Investig Bioméd. 2014;33(4):405-13.

17. De Marco R, Ceccarelli R, Frulio R, Palmero C, Vittone P. Retinochoroiditis associated with congenital toxoplasmosis in children: IgG antibody profiles demonstrating the synthesis of local antibodies. Eur J Ophthalmol. 2003;13(1):74-9.

18. Ospina O S. Diagnóstico de la infección por el virus de la inmunodeficiencia humana Diagnosis of human immunodeficiency virus. Infectio. 2006;10(4):273-8.

19. Boothroyd JC. Toxoplasma gondii: 25 years and 25 major advances for the field. Int $\mathrm{J}$ Parasitol. 2009;39(8):935-46.
20. Murat J-B, L'Ollivier $C$, Fricker Hidalgo $H$, Franck J, Pelloux H, Piarroux R. Evaluation of the New Elecsys Toxo IgG Avidity Assay for Toxoplasmosis and New Insights into the Interpretation of Avidity Results. Clin Vaccine Immunol CVI. 2012;19(11):183843.

21. Marcolino PT, Silva DAO, Leser PG, Camargo ME, Mineo JR. Molecular Markers in Acute and Chronic Phases of Human Toxoplasmosis: Determination of Immunoglobulin G Avidity by Western Blotting. Clin Diagn Lab Immunol. 2000;7(3):384-9.

22. Rilling V, Dietz K, Krczal D, Knotek F, Enders G. Evaluation of a commercial IgG/IgM Western blot assay for early postnatal diagnosis of congenital toxoplasmosis. Eur J Clin Microbiol Infect Dis Off Publ Eur Soc Clin Microbiol. 2003;22(3):174-80.

23. Bradford MM. A rapid and sensitive method for the quantitation of microgram quantities of protein utilizing the principle of proteindye binding. Anal Biochem. $1976 ; 72(1): 248-54$.

24. Laemmli UK. Cleavage of Structural Proteins during the Assembly of the Head of Bacteriophage T4. Nature. 1970;227(5259):680-5.

25. Towbin H, Staehelin T, Gordon J. Electrophoretic transfer of proteins from polyacrylamide gels to nitrocellulose sheets: procedure and some applications. Proc Natl Acad Sci U S A. 1979;76(9):4350-4.

26. Khammari I, Lakhal $S$, Westermann $B$, Benkahla A, Bouratbine A, Dorsselaer AV, et al. Characterization of Soluble and Membrane-Bound Proteins of Toxoplasma gondii as Diagnostic Markers of Infection. ] Bacteriol Parasitol [Internet]. 2015 [citado 27 de febrero de 2018]; 06(04). Disponible en: https://www.omicsonline.org/openaccess/characterization-of-soluble-andmembranebound-proteins-of-toxoplasmagondii-as-diagnostic-markers-of-infection2155-9597-1000239. php?aid=58651

27. Mohammadpour N, Saki J, Rafiei A, Khodadadi A, Tavalla M. Evaluating the Antigenic Markers of Toxoplasma gondii $\mathrm{RH}$ strain for Differential Diagnosis of Acute and Chronic Toxoplasmosis. Int J Pharm Res Allied Sci. 2016;5:429-35.

28. Ahn $M H$, Son $H J$, Leem $M H$, Min DY. [Antigen analysis of Toxoplasma gondii Iysate and excretory-secretory materials by enzyme-linked immunoelectrotransfer blot (EITB)]. Korean J Parasitol. 1994;32(4):249-57.

29. Garweg JG, Garweg S-DL, Flueckiger F, Jacquier P, Boehnke M. Aqueous Humor and Serum Immunoblotting for Immunoglobulin Types G, A, M, and $E$ in Cases of Human Ocular Toxoplasmosis. J Clin Microbiol. 2004;42(10):4593-8. 
30. Daryani A, Sharif M, Kalani $H$, Rafiei A, Kalani F, Ahmadpour E. Electrophoretic Patterns of Toxoplasma gondii Excreted/Secreted Antigens and Their Role in Induction of the Humoral Immune Response. Jundishapur J Microbiol. 2014;7(4):e9525-e9525.

31. Ali-Heydari S, Keshavarz H, Shojaee S, Mohebali M. Diagnosis of antigenic markers of acute toxoplasmosis by IgG avidity immunoblotting. Parasite [Internet]. 2013 [citado 22 de noviembre de 2018];20.

Disponible

en:

https://www.ncbi.nlm.nih.gov/pmc/articles/ PMC3718541/

32. Stroehle A, Schmid K, Heinzer I, Naguleswaran A, Hemphill A. Performance of a Western immunoblot assay to detect specific anti-Toxoplasma gondii IgG antibodies in human saliva. J Parasitol. 2005;91(3):561-3. 